\title{
USO DO GLICOSÍMETRO PORTÁTIL PARA DETERMINAR A CONCENTRAÇÃO DE GLICOSE NO SANGUE DE CÃES
}

\author{
Grazielle Sousa Aleixo, ${ }^{1}$ Maria Cristina Oliveira Coelho, ${ }^{2}$ Ana Paula Monteiro Tenório, ${ }^{3}$ \\ Ana Luiza Neves Guimarães, ${ }^{4}$ Moacir Bezerra Andrade ${ }^{5}$ e Humberto Barros Cavalcanti ${ }^{6}$
}

\footnotetext{
1. Departamento de Medicina Veterinária (DMV) da UFRPE, área de cirurgia e clínica de pequenos animais. E-mail: grazielle@yahoo.com 2. DMV, área de cirurgia e clínica de pequenos animais

3. DMV, área de anestesiologia e terapêutica veterinária

4. DMV, área de clínica de pequenos animais

5. DMV, área de clínica de pequenos animais 6. Médico veterinário autônomo.
}

\section{RESUMO}

Uma das maneiras de monitorar a concentração de glicose no sangue é através de um aparelho portátil chamado glicosímetro. O equipamento, desenvolvido para mensurar a glicemia de pacientes humanos, foi empregado neste experimento para verificar se os resultados com amostras de sangue de cães também são confiáveis. Para realizar o exame, foi colhida uma gota de sangue capilar da orelha de 36 caninos. A variação média entre o glicosímetro em comparação com o método laboratorial de referência foi de 7,90 \%, estando este valor dentro dos padrões estabelecidos por órgãos reguladores da área, como o American Diabetes Association (ADA). Conclui-se que o glicosímetro é uma ferramenta confiável para dosar e/ou monitorar a glicemia em cães, tendo como vantagens a praticidade e rapidez com que os resultados são fornecidos.

PALAVRAS-CHAVES: Cão, glicemia, monitoramento.

\section{ABSTRACT}

\section{USE OF THE PORTABLE GLUCOMETER TO DETERMINE GLUCOSE CONCENTRATION IN DOGS' BLOOD}

One of the ways of monitoring the glucose concentration in the blood is with a portable device, known as glucometer. The equipment, developed to measure the glycemia of human patients, was used in this experiment with the purpose of verifying if the results of samples of dogs' blood are also reliable. To perform the exam a drop of capillary blood from the ear of 36 dogs was collected. The mean variation when comparing the glucometer and the

KEYWORDS: Dog, glycemia, monitoring.

\section{INTRODUÇÃO}

A glicose é um carboidrato constantemente utilizado pelo organismo como fonte de energia (BUSH, laboratorial reference method was $7.90 \%$, being this result within the patterns established by regulatory organs of the area, such as the American Diabetes Association (ADA). It was concluded that the portable glucometer is a reliable tool to dose and/or monitor dogs glycemia, having as advantages the practicality and speed for obtaining the results.

2004), entretanto doenças graves como, por exemplo, a Diabetes mellitus, podem ocasionar estados de hiper ou hipoglicemia que, se não forem diagnosticados e tratados com rapidez e adequadamente, poderão gerar 
sérias complicações no estado de saúde do paciente, inclusive levando à sua morte (NELSON, 1997; HERRTAGE, 2001; PICA et al., 2003).

A determinação da concentração de glicose e as curvas glicêmicas seriadas são importantes no manejo a longo termo da diabetes em cães e gatos (CASELLA et al., 2002; CASELLA et al., 2003). Os resultados da monitoração são usados para avaliar a eficácia do tratamento e fazer ajustes na dieta e prescrições médicas (ex.: dosagem de insulina) e, assim, atingir o melhor controle da glicose sanguínea (LARIN et al., 2002; PICA et al., 2003; GOLDSTEIN et al., 2004; PICKUP et al., 2005).

Existem diferentes maneiras de se mensurar a glicemia (COHN et al., 2000; PEREIRA et al., 2003;
KUMAR et al., 2004), sendo o glicosímetro portátil um dos métodos recomendados pelo College of American Pathologists (PASCALI, 2004). Na medicina humana essa técnica é bastante empregada, por se tratar de um método fácil, de custo relativamente baixo e que fornece os resultados com rapidez (COHN et al., 2000; WESS; REUSCH, 2000a; STEIN \& GRECO, 2002).

Os glicosímetros oferecem uma série de benefícios em relação aos analisadores automáticos-padrões, utilizados em laboratórios de diagnóstico ( $\mathrm{COHN}$ et al., 2000) (Quadro 1), entre eles o fato de serem pequenos, portáteis, fáceis de manusear, além de requererem uma pequena quantidade de sangue para se realizar o exame (FOSTER et al., 1999; COHN et al., 2000; WESS \& REUSCH, 2000a; WESS \& REUSCH, 2000b).

QUADRO 1. Algumas características do glicosímetro portátil e método laboratorial para mensurar a glicemia, em relação ao custo, tamanho da amostra e do equipamento, tempo de realização do teste e resultado fornecido

\begin{tabular}{|l|c|c|}
\hline & Glicosímetro portátil & Método laboratorial \\
\hline Custo por análise & Menor & Maior \\
\hline Tamanho da amostra & Menor (entre 3 a $5 \mu \mathrm{L})$ & Maior $(3 \mathrm{~mL})$ \\
\hline Equipamento & Portátil & portátil \\
\hline Tempo de realização & Mais rápido $(20$ a 60 segundos $)$ & Mais confiável \\
\hline Resultado & Susceptível a maiores erros & 2 horas $)$ \\
\hline
\end{tabular}

Muitos profissionais da área de saúde mensuram os níveis de glicose sanguínea dos seus pacientes com glicosímetros aprovados para uso doméstico, ao invés de utilizar os métodos laboratoriais, que são mais caros e demoram mais tempo para serem realizados (GOLDSTEIN et al., 2004).

A maioria dos monitores portáteis de glicose foi desenvolvida para amostras de sangue capilar (FUNK et al., 2001; CASELLA, 2003; KERR, 2003; KUMAR et al., 2004; NEWMAN \& TURNER, 2005). A primeira geração de aparelhos utilizava a mensuração fotométrica, baseada em uma mudança de cor na tira de teste provocada pela reação do sangue com uma enzima contida nela (BRIGGS \& CORNELL, 2004), sendo a intensidade da cor proporcional à quantidade de glicose (AMERICAN DIABETES ASSOCIATION, 1996). Os glicosímetros utilizados atualmente quantificam a glicose através de reações eletroquímicas (PICA et AL., 2003; PASCALI, 2004), que geram um impulso elétrico a ser interpretado pelo aparelho, como a concentração de glicose na amostra (FOSTER et al., 1999; PICA et al., 2003).

A popularização do controle domiciliar da glicemia em pacientes humanos diabéticos resultou no desenvolvimento e lançamento de vários glicosímetros (FRISHMAN et al., 1992; KERR, 2003), que diferem em relação à quantidade de sangue necessário para realizar o teste, velocidade com que o resultado é fornecido, tamanho do aparelho, capacidade em armazenar resultados, tecnologia que emprega, custo e tipo de tira de teste utilizada (BRIGGS \& CORNELL, 2004).

As melhorias alcançadas nos últimos anos incluem maior precisão, mensurações mais rápidas (WEITGASSER et al., 1999; WESS \& REUSCH, 2000a) e facilidade de uso (AMERICAN DIABETES ASSOCIATION, 1996; BÖHME et al., 2003), além de pequena quantidade de sangue para análise 
(WIENER, 2000; CORNELL, 2003; BRIGGS \& CORNELL, 2004; NEWMAN \& TURNER, 2005). Como os resultados obtidos por esses equipamentos vão frequentemente orientar o tratamento clínico a ser instituído no paciente, é muito importante que eles forneçam informações precisas (COHN et al., 2000).

Na medicina veterinária são raros os trabalhos que se referem ao uso e acurácia dos resultados alcançados pelo glicosímetro em relação aos métodos utilizados em laboratórios, que são considerados padrão. Levando essa observação em consideração, o presente trabalho tem por objetivo descrever o uso do monitor portátil para dosar a concentração de glicose no sangue de cães, avaliando a eficiência do exame.

\section{MATERIAL E MÉTODOS}

Para o desenvolvimento da pesquisa foram utilizados 36 animais da espécie canina, machos e fêmeas, de raças, pesos e idades variadas, atendidos no Hospital Veterinário (HV) do Departamento de Medicina Veterinária (DMV) da Universidade Federal Rural de Pernambuco (UFRPE).

A dosagem da concentração de glicose sérica foi realizada como parte integrante dos testes de rotina durante o exame clínico do paciente, usando-se, para tal procedimento, um aparelho portátil de glicemia ${ }^{\mathrm{a}}$. Todos os pacientes estavam de jejum por um período aproximado de oito a doze horas.

Foi coletada uma amostra de sangue capilar através de uma punção na face medial da orelha externa do animal produzida por uma agulha hipodérmica descartável ${ }^{\mathrm{b}}$ calibre $25 \times 0,7 \mathrm{~mm}$, após tricotomia e antissepsia da região com solução de álcool a $70 \%$ c, como recomendado pelo fabricante do produto. No prazo máximo de trinta segundos após a punção, a tira de teste ${ }^{\mathrm{d}}$ era inserida no equipamento, que nesse momento ligava automaticamente. Era necessário verificar se o código apresentado no visor do glicosímetro correspondia ao número fornecido no tubo de tiras de teste, para que a calibração do aparelho fosse efetuada (Figura 1).

Ao aparecer um símbolo em forma de gota no monitor, aplicava-se a gota de sangue gerada pela punção na curva de conforto da tira de teste (faixa amarela) (Figura 2).

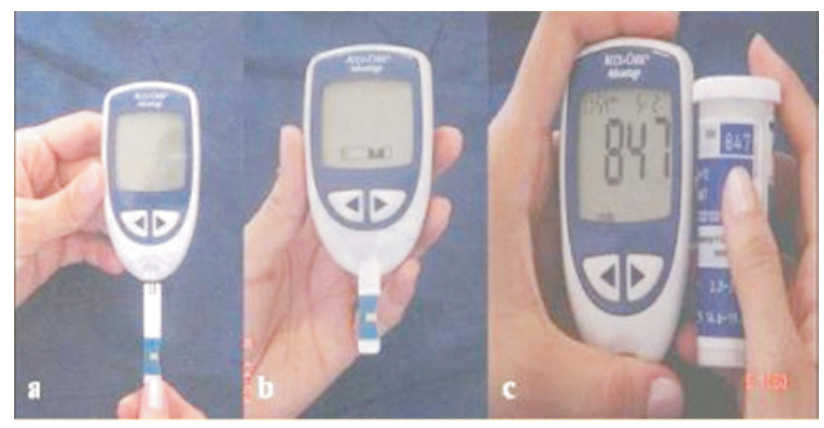

FIGURA 1. a) Tira de teste sendo inserida no monitor desligado; b) O monitor liga automaticamente; c) O número que aparece no visor do glicosímetro corresponde ao da embalagem das tiras de teste.

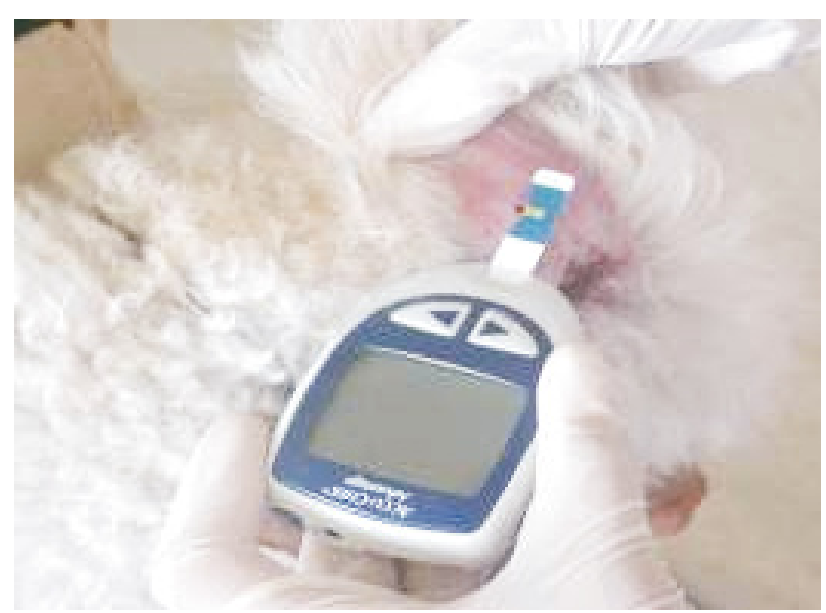

FIGURA 2. Visualização da amostra de sangue capilar sendo aplicada na curva de conforto da tira de teste

Após 26 segundos, era feita a leitura da concentração de glicose sanguínea em $\mathrm{mg} / \mathrm{dL}$ expressa no painel de leitura do aparelho. Depois da realização do teste, a tira era removida do aparelho e descartada em local apropriado. O equipamento era limpado e armazenado juntamente com o tubo de fitas reagentes, de acordo com as recomendações do fabricante.

Com o intuito de comparar os resultados obtidos no glicosímetro com um método de referência, também foi coletada, em cada paciente, uma amostra de três $\mathrm{mL}$ de sangue venoso para ser submetido ao teste de glicemia, segundo o método enzimático-colorimétrico GOD-POD, descrito por TRINDER (1969).

A amostra, colhida por punção da veia cefálica ou safena, era acondicionada em um tubo de ensaio contendo uma gota de etileno diamino tetra-acético (EDTA) com fluoreto de sódio ${ }^{\mathrm{e}}$ (conservante de glico- 
se) e imediatamente centrifugada a 2.500 rotações por minuto (rpm) por dez minutos, para obtenção do plasma. Em seguida, transferia-se o plasma para um tubo de eppendorf, devidamente identificado e refrigerado a $4^{\circ} \mathrm{C}$, para posteriormente ser submetido ao teste de glicose em um prazo máximo de 72 horas.

Para realização do exame laboratorial, foi empregado o kit de glicose $\mathrm{SL}^{\mathrm{f}}$. Uma alíquota de $10 \mu \mathrm{L}$ da amostra de plasma era adicionada a $1 \mathrm{~mL}$ do reativo de trabalho em um tubo de ensaio e incubada em banho-maria a $37^{\circ} \mathrm{C}$ por dez minutos. Decorrido esse tempo, retirava-se a amostra do banho e a coloração rósea desenvolvida era lida em espectrofotômetro a
$505 \mathrm{~nm}$ no sistema de bioquímica semiautomáticog (Figura 3).

Com o intuito de certificar que o tamanho da amostra foi suficiente, empregou-se o processo amostral inteiramente aleatório ao nível de 5\% de probabilidades e um erro adotado de 5\% (SCHEAFFER et al., 1979). E para verificar se existia diferença significativa entre os resultados de glicemia alcançados com o glicosímetro portátil e o método enzimático-colorimétrico GODPOD, descrito por TRINDER (1969), considerou-se um delineamento inteiramente aleatório ao nível de $1 \%$ de probabilidades (SAMPAIO, 1998).
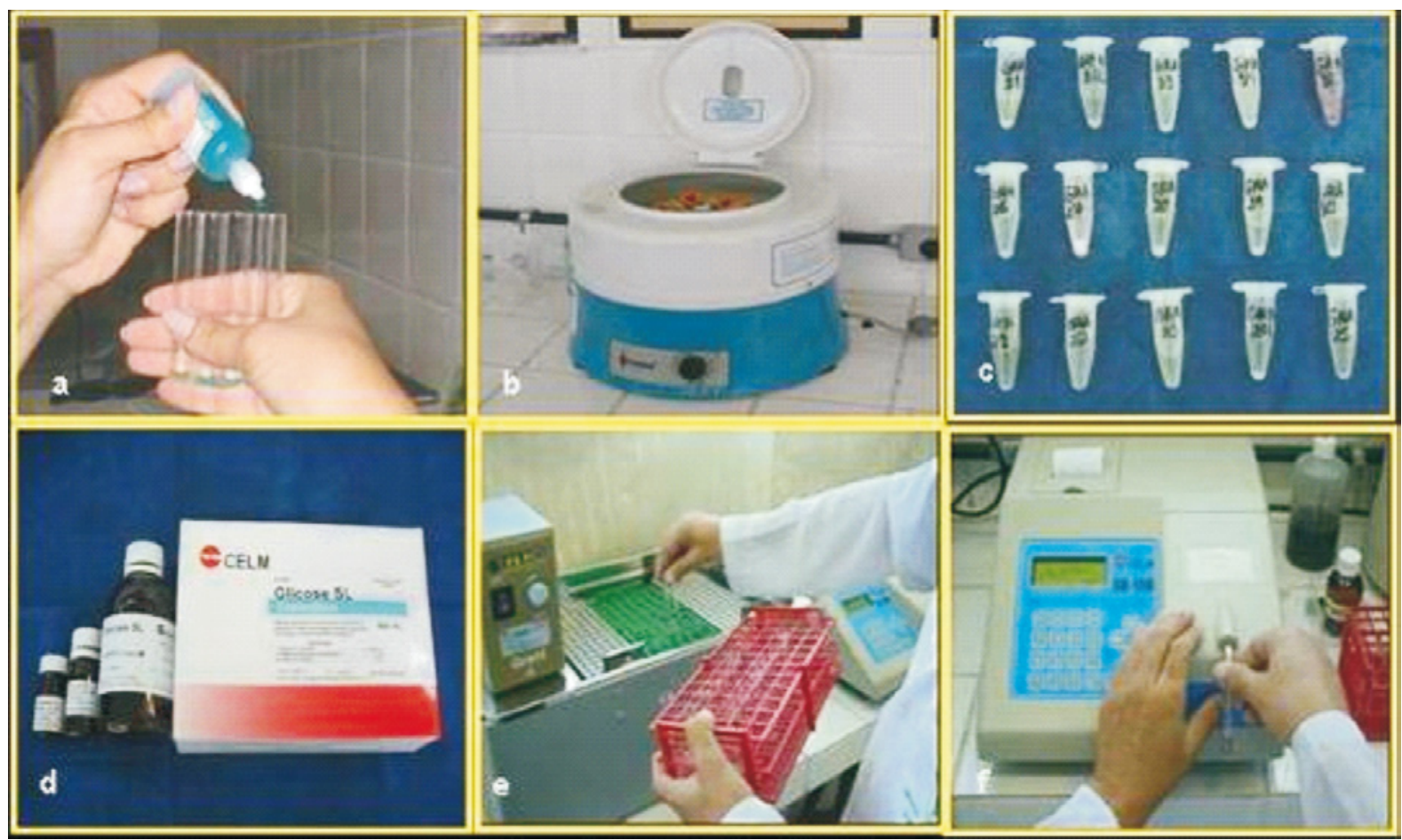

FIGURA 3. a) Adição do EDTA fluoretado nos tubos de ensaio; b) Centrífuga utilizada para obtenção do plasma; c) Tubos de eppendorf contendo as amostras de plasma; d) Kit reagente empregado para determinação da glicose pelo método laboratorial; e) Amostras sendo colocadas em banho-maria; f) Aspiração de uma amostra pelo aparelho de bioquímica semiautomático.

\section{RESULTADOS E DISCUSSÃO}

A concentração de glicose dos 36 animais no aparelho portátil variou entre 60 e $95 \mathrm{mg} / \mathrm{dL}$, enquanto que no método laboratorial essa variação foi de 62 a $106 \mathrm{mg} / \mathrm{dL}$. Os valores de glicemia para cada método estão representados no Gráfico 1.
De acordo com CASELLA(2002), vários glicosímetros portáteis têm sido avaliados e tem se constatado que os resultados fornecidos são próximos dos obtidos com o método de referência ao se empregar amostras de sangue humano, corroborando os resultados alcançados neste estudo com cães em que não houve diferença estatística significativa entre os diferentes métodos. 


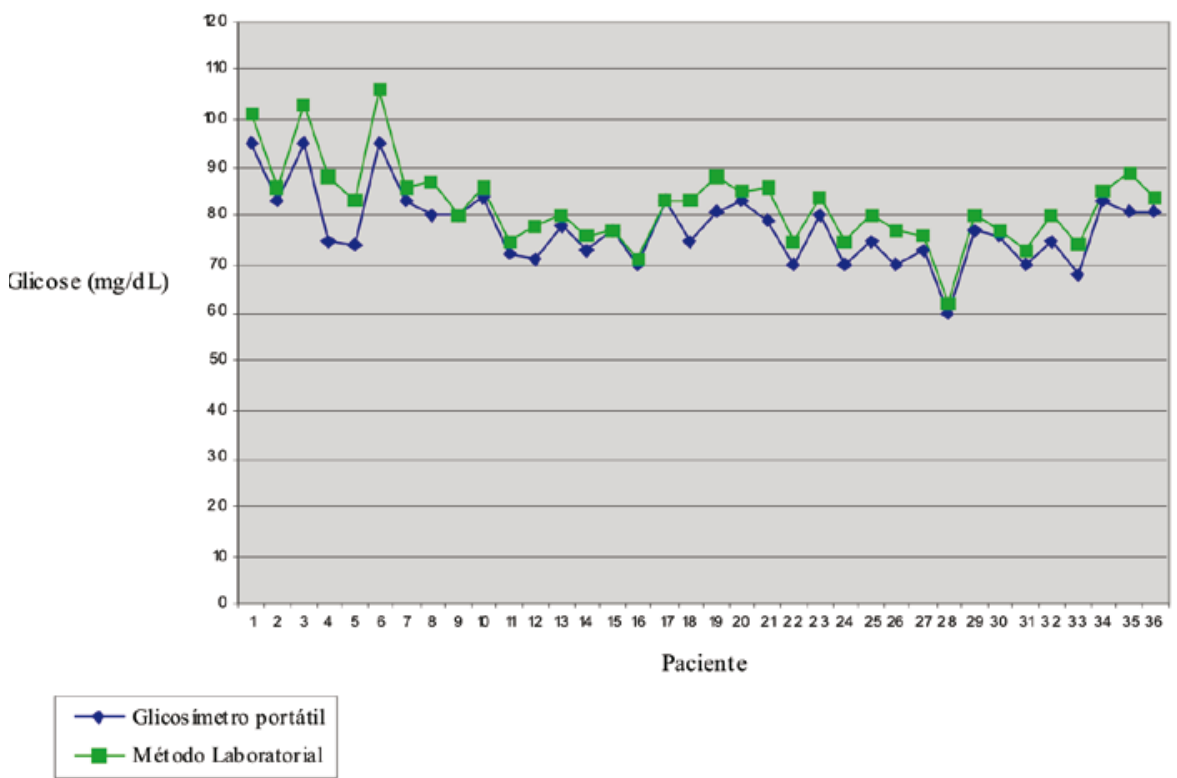

GRÁFICO 1. Concentração de glicose em mg/dL de 36 cães, alcançados no glicosímetro portátil e pelo método laboratorial.

No presente estudo, todas as amostras analisadas se encontravam dentro dos padrões estabelecidos pela ADA em 1986, propondo-se que as mensurações realizadas com o glicosímetro portátil podem ser diferentes em até $15 \%$ do valor de referência (JOHNSON \& BAKER, 2001). A Food and Drug Administration (FDA) requer que todos os glicosímetros tenham uma taxa de erro $<20 \%$ na concentração de glicose sanguínea para valores entre 30 e $400 \mathrm{mg} / \mathrm{dL}$ (BRIGGS \& CORNELL, 2004). Já a International Organization for Standardization (ISO) recomendou, em 2001, que 95\% das mensurações devem estar entre +- $20 \%$ dos valores de referência para concentrações de glicose $>$ $100 \mathrm{mg} / \mathrm{dL}$ e entre +- $20 \mathrm{mg} / \mathrm{dL}$ para valores de glicose $<100$ mg/dL (DAI et al., 2004). Como a variação média entre os dois métodos empregados foi de 7,90\%, percebe-se que esse valor se encontra dentro das metas preconizadas por todas as entidades anteriormente citadas. Os resultados para as estatísticas descritivas estão na Tabela 1.

TABELA 1. Média aritmética da glicemia alcançada em cada método $(\overline{\mathrm{X}})$, desvio padrão $\left(\mathrm{s}_{\mathrm{x}}\right)$, coeficiente de variação $(\mathrm{CV} \%)$, número de amostras representativo da população (NAR) e erro de amostragem

\begin{tabular}{ccc}
\hline & Glicosímetro & Método laboratorial \\
\hline$\overline{\mathrm{X}}$ & $76.06 \mathrm{mg} / \mathrm{dL}$ & $82.53 \mathrm{mg} / \mathrm{dL}$ \\
$\mathbf{s}_{\mathbf{x}}$ & 7.2 & 8.6 \\
$\mathrm{CV} \%$ & 9.4 & 10.4 \\
$\mathrm{NAR}$ & 15 & 18 \\
$\mathrm{EA} \%$ & 4.4 & 4.9 \\
\hline
\end{tabular}

Observa-se que, em ambos os métodos, o número da amostra foi representativo, isto é, utilizou-se um maior número de animais que o mínimo requerido para representar os métodos, o que, consequentemente, proporcionou erros de amostragem inferiores ao adotado (5\%). 
Nas primeiras pesquisas realizadas em 1988 e 1991, nenhum dos monitores avaliados atingiu as metas estabelecidas pela ADA em 1986 (JOHNSON \& BAKER, 2001). Os avanços tecnológicos alcançados pelas empresas fabricantes de glicosímetros nos últimos anos permitiram que os novos modelos se tornassem mais fáceis de manusear e, consequentemente, as avaliações mais recentes têm indicado vários aparelhos com o potencial de atingir o total de erro de $15 \%$ do método de referência (JOHNSON \& BAKER, 2001), como aconteceu com o glicosímetro empregado neste experimento.

Em um estudo realizado em 2004, utilizando novamente sangue de seres humanos, constatou-se a existência de uma pequena diferença entre os resultados obtidos no glicosímetro com relação ao método laboratorial (KUMAR et al., 2004), sendo considerada sem importância clínica. Da mesma maneira, a mínima diferença percebida entre os métodos utilizados nesta pesquisa não permitiu que nenhum cão tivesse um resultado além dos valores de referência de glicemia para a espécie (60 a $125 \mathrm{mg} / \mathrm{dL}$ : TILLEY \& SMITH, 2003) no glicosímetro, com um padrão normal no teste laboratorial, ou vice-versa.

Em contraste com os resultados obtidos em uma pesquisa na qual a maioria dos glicosímetros avaliados fornecia valores mais altos ou mais baixos das concentrações de glicose (WESS \& REUSCH, 2000 b), o modelo usado no presente estudo constantemente indicava uma concentração de glicose mais baixa quando comparado ao método-padrão. Isso é considerado uma vantagem, porque os erros se tornam mais previsíveis (CASELLA, 2003; CASELLA et al., 2003). A menor concentração de glicose sanguínea consistentemente obtida pelo glicosímetro é explicada pelo fato de a glicemia em sangue total ser entre $10 \%$ e $15 \%$ mais baixa que no plasma (PICA et al., 2003). A razão para essa diferença está no fato de o conteúdo de glicose no eritrócito ser menor que no plasma, dada a natureza densa dessa célula (BRIGGS \& CORNELL, 2004).

Como citado anteriormente, a facilidade no uso do monitor portátil tem aumentado a precisão dos exames, pois erros provocados pelo usuário são a primeira causa de falta de acurácia nos resultados (BRIGGS \& CORNELL, 2004), como a aplicação inapropriada da amostra e a ultrapassagem do limite de tempo para realização do exame (COHN et al., 2000; BÖHME et al., 2003). Algumas dessas falhas foram observadas em um estudo realizado por ALEIXO et al. (2006), em que algumas amostras foram consideradas impróprias para análise, pelo fato de a aplicação do sangue na tira de teste não ter sido adequada ou o tempo necessário para a realização do teste ter ultrapassado.

Além desses erros, outras fontes de falha são a utilização de reagentes defeituosos, glicosímetros não calibrados e também o não armazenamento do glicosímetro ou tiras de teste de acordo com as recomendações do fabricante (BRIGGS \& CORNELL, 2004). Recomenda-se armazenar adequadamente as fitas reagentes e o aparelho, fazer a calibração do equipamento, utilizar regularmente as soluçõescontroles, colocar a quantidade correta de sangue na tira de teste e manter o aparelho sempre limpo. No local onde se deseja realizar a punção para a coleta da amostra, é preciso ter cuidado com a higiene, limpando a área com água e sabão ou solução de álcool a 70\%, após a tricotomia (PASCALI, 2004). Todas essas recomendações foram seguidas como descrito em material e métodos, com o intuito de reduzir ao máximo a possibilidade de alterações no resultado por erro do usuário.

O responsável pela execução dos exames também foi submetido a um treinamento prévio. $O$ treino apropriado para utilizar a tecnologia do glicosímetro é um fator decisivo para reduzir as falhas relacionadas ao usuário. Ele é necessário não somente para os indivíduos diabéticos que realizam a automonitoração, mas também para profissionais da área de saúde que usam o monitor para dosar a glicemia de seus pacientes (NSCQA, 1993).

A rapidez com que os resultados são obtidos é outro fator que deve ser levado em consideração, no momento de decidir que tipo de método adotar (PICA et al., 2003). É relatado que, especialmente durante os procedimentos cirúrgicos, é importante que o nível de glicose no sangue seja mantido na normalidade e que devem ser evitados estados de hipo ou hiperglicemia transoperatórios (AUSTIN et al., 2000). Como o equipamento utilizado neste estudo fornece o resultado em aproximadamente quarenta segundos, sugere-se a sua utilização, ao invés do método laboratorial, para dosar a glicemia durante cirurgias.

Também tem sido descrito que os mais novos glicosímetros precisam de apenas 3 a $5 \mu \mathrm{L}$ de sangue 
para a execução do exame, o que é uma considerável redução, em comparação com os modelos antigos, que necessitavam de 10 a $50 \mu \mathrm{L}$, diminuindo o risco da ocorrência de erros na aplicação da gota de sangue na tira de teste (WEITGASSER et al., 1999). Em relação aos pacientes caninos acompanhados no experimento, percebeu-se que isso facilitou a realização do procedimento, pelo fato de a coleta ter sido mais rápida, do que se tivesse de ser usada uma amostra maior, como nos monitores mais antigos. Ao mesmo tempo, isso permite conseguir uma amostra representativa de sangue com o mínimo de dor (NEWMAN \& TURNER, 2005), o que foi constatado nesta pesquisa, visto que nenhum dos pacientes demonstrou dor ou incômodo com a punção realizada na orelha. De acordo com MIRA et al. (2006), a dor apresentada pelo paciente no momento da realização do exame pode se tornar um fator limitante para o uso do glicosímetro. Outra consideração importante é que coletar a amostra sem estressar o paciente é um beneficio, porque previne uma hiperglicemia transitória (SILVA JÚNIOR et al., 2005). Com o intuito de evitar esse quadro hiperglicêmico temporário, durante as coletas se evitou submeter o paciente ao estresse, procurando contê-lo com tranquilidade, manipulando a região da coleta com delicadeza e realizando o exame com rapidez.

O glicosímetro também é o método mais indicado para mensurações em amostras de sangue lipêmicas ou hemolisadas, pelo fato de a opacidade interferir nos métodos laboratoriais (KERR, 2003), com tipos de amostras que podem levar a resultados equivocadamente elevados no exame laboratorial (BUSH, 2004). Tendo em vista que pacientes encaminhados para atendimento clínico podem apresentar quadros de hemólise, icterícia ou lipemia ocasionados por diversas afecções, o glicosímetro portátil passa a ser uma boa alternativa para essas situações. Durante a realização do experimento, além das 36 amostras avaliadas, outras oito não foram consideradas para a análise estatística. Isso porque, depois de centrifugadas para realização do teste laboratorial, duas estavam hemolisadas, quatro se encontravam ictéricas e mais duas apresentavam aspecto leitoso. Os resultados alcançados com essas amostras foram em média $40 \%$ superiores aos do glicosímetro portátil, confirmando, assim, que esse tipo de amostra pode levar a resultados falsamente elevados nas análises laboratoriais.

\section{CONCLUSÕES}

O glicosímetro portátil, ao ser utilizado seguindo todas as recomendações do fabricante, demonstrou precisão na determinação dos níveis glicêmicos em cães. Trata-se de um instrumento que apresenta como vantagens o fato de ser um teste de fácil execução e de fornecer os resultados em poucos segundos.

\section{MATERIAL UTILIZADO}

a) Glicosímetro portátil Accu-Chek ${ }^{\circledR}$ Advantage - Roche Diagnóstica Brasil Ltda., São Paulo, SP

b) Agulha hipodérmica descartável - Plascalp, Feira de Santana, BA

c) Álcool a 70\% - Miyako do Brasil Ind.

Com. Ltda., Guarulhos, SP

d) Tiras de teste Accu-Chek ${ }^{\circledR}$ Advantage II -

Roche Diagnóstica Brasil Ltda., São Paulo, SP

e) EDTA com fluoreto de sódio - GLISTAB ${ }^{\circledR}$

- Labtest, Lagoa Santa, MG

f) Kit de Glicose SL - CELM, Barueri, SP

g) Sistema de bioquímica semiautomático modelo SB 190 - CELM, Barueri, SP.

\section{REFERÊNCIAS}

ALEIXO, G. A. S.; COELHO, M. C. O. C.; GUIMARÃES, A. L. N.; ANDRADE, M. B.; LOPES JÚNIOR, C. G.; SILVA, R. A. A.; CAVALCANTI, H. B. Fatores que podem invalidar os resultados da mensuração dos níveis glicêmicos em cães utilizando o glicosímetro portátil. Ciência Animal Brasileira, v. 7, n. 4, p. 447-454, 2006.

AMERICAN DIABETES ASSOCIATION. Self-monitoring of blood glucose (Consensus Statement). Diabetes Care, v. 19 (1S), p. 62S-66S, 1996.

AUSTIN, S; MCATAMNEY, D.; MAGUIRE, P.; LOAN, P.; CREAN, P. M. A study to assess the feasibility of using glucometer measurement of blood glucose on fresh blood sampled from the surgical field. Paediatric Anaesthesia, v. 10, p. 691-692, 2000.

BÖHME, P.; FLORIOT, M.; SIRVEAUX, M.; DURAIN, D.; ZIEGLER, O.; DROUIN, P.; GUERCI, B. Evolution of analytical performance in portable glucose meters in the last decade. Diabetes Care, v. 26, n. 4, p. 1170-1175, 2003.

BRIGGS, A. L.; CORNELL, S. Self-monitoring blood glucose (SMBG): now and the future. Journal of Pharmacy Practice, v. 17, p. 29-38, 2004. 
BUSH, B. M. Nutrientes e Metabólitos. In: Interpretação de resultados laboratoriais para clínicos de pequenos animais. São Paulo: Roca, 2004. p. 167-223.

CASELLA, M.; WESS, G.; REUSCH, C. E. Measurement of capillary blood glucose concentrations by pet owners: a new tool in the management of diabetes mellitus. Journal of the American Animal Hospital Association, v. 38, p. 239-245, 2002.

CASELLA, M.; WESS, G.; HASSIG, M.; REUSCH, C. E. Home monitoring of blood glucose concentrations by owners of diabetes dogs. Journal of Small Animal Practice, v. 44, p. 298-305, 2003.

COHN, L. A.; MCCAW, D. L.; TATE, D. J.; JOHNSON, J. C. Assessment of five portable blood glucose meters, a point-of-care analyzer, and color test strips for measuring blood glucose concentration in dogs. Journal of the American Veterinary Medical Association, v. 216, n. 2, p. 198-202, 2000.

CORNELL, S. Self-monitoring blood glucose monitors update. Advances in Pharmacy, v. 1, p. 106-111, 2003.

DAI, K.; TAI, D.; HO, P.; CHEN, C.; PENG, W.; CHEN, S.; HSU, C.; LIU, Y.; HSIEH, H.; YANG, C.; TSAI, M.; MAO, S. J. T. Accuracy of the easy touch blood glucose self-monitoring system: a study of 516 cases. Clinic Chimica Acta, v. 349, p. 135-141, 2004.

FOSTER, S. A.; GOODE, J. K. R.; SMALL, R. E. Home blood glucose monitoring. The Annals of Pharmacotherapy, v. 33, p. 355-363, 1999.

FRISHMAN, D.; ARDITO, D.; GRAHAM, S. M. Performance of glucose monitors. Laboratory Medicine, v. 23, n. 3, p. 179184, 1992.

FUNK, D. L.; CHAN, L.; LUTZ, N.; VERDILE, V. P. Comparison of capillary and venous glucose measurements in healthy volunteers. Prehospital Emergency Care, v. 5, n. 3, p. 275-277, 2001.

GOLDSTEIN, D. E.; LITTLE, R. R.; LORENZ, R. A.; MALONE, J. L.; NATHAN, D.; PETERSON, C. M.; SACKS, D. B. Teste of glycemia in diabetes. Diabetes Care, v. 27, n. 7, p. 1761-1773, 2004.

HERRTAGE, M. E. Doenças do sistema endócrino. In: DUNN, J. $\mathrm{K}$. Tratado de medicina de pequenos animais. São Paulo: Roca, 2001. p. 523-568.

JOHNSON, R. N.; BAKER, J. R. Erro detection and measurement in glucose monitors. Clinica Chimica Acta, v. 307, p. 61-67, 2001.

KERR, M. G. Exames práticos na clínica. In: Exames laboratoriais em medicina veterinária (bioquímica clínica e hematologia). 2. ed. São Paulo: Roca, 2003. p. 327-338.
KUMAR, G.; LEONG, B.; KUMAR, S. Correlation of capillary and venous blood glucometry with laboratory determination. Prehospital Emergency Care, v. 8, n. 4, p. 378-383, 2004.

LARIN, K. V.; ELEDRISSI, M. S.; MOTAMEDI, M.; ESENALIEV, R. O. Noninvasive blood glucose monitoring with optical coherence tomography. Diabetes Care, v. 25, n. 12, p. 2263-2267, 2002.

MIRA, G. S.; CANDIDO, L. M. B.; YALE, J. F. Performance de glicosímetro utilizado no automonitoramento glicêmico de portadores de diabetes mellitus tipo 1. Arquivos Brasileiros de Endocrinologia \& Metabologia, v. 50, n. 3, p. 541-549, 2006.

NELSON, R. W. Diabete melito. In: ETTINGER, S. J.; FELDMAN, E. C. Tratado de medicina interna veterinária II. v. 4. ed. São Paulo: Manole, 1997. p. 2085-2122.

NEWMAN, J. D.; TURNER, A. P. F. Home blood glucose biosensors: a comercial perspective. Biosensors and Bioelectronics, v. 20, p. 2435-2453, 2005.

NSCQA - The National Sttering Committee for Quality Assurance In Capillary Blood Glucose Monitoring). Proposed strategies for reducing user error in capillary blood glucose monitoring. Diabetes Care, v. 16, n. 2, p. 493-498, 1993.

PASCALI, P. M. Monitorização da glicemia capilar. BD Terapêutica em Diabetes, ano 9, n. 31, p. 4-5, 2004.

PICA, C. Q.; MENEZES, J. R.; ALBERTAZZI, J. A.; CAMIÑA, R. M. Avaliação comparativa de glicosímetros portáteis através de curva glicêmica induzida. In: CONGRESSO BRASILEIRO DE METROlOGIA, 3., 2003, Recife. Anais .... Recife: SBM, 2003. p. 1-7. Disponível em: <www.metrologia2003.org.br/anais_congresso/MA0365.pdf>

PICKUP, J. C.; HUSSAIN, F.; EVANS, N. D.; SACHEDINA, N. In vivo glucose monitoring: the clinical reality and the promise. Biosensors and Bioelectronics, v. 20, p. 1897-1902, 2005.

SAMPAIO, I. B. M. Estatística aplicada à experimentação animal. Minas Gerais: UFMG, 1998. 221 p.

SCHEAFFER, R. L.; MENDENHALL, W.; OTT, L. Elementary survey sampling. Boston: Duxbury, 1979. 278 p.

SILVA JÚNIOR, J. W.; BORGES, F. M. O.; MURGAS, L. D. S.; VALÉRIO, A. G.; MEDEIROS, G. C.; VIANA, R.; LIMA, L. M. $\mathrm{S}$. Digestibilidade de dietas com diferentes fontes de carboidratos e sua influência na glicemia e insulinemia de cães. Ciência e Agrotecnologia, v. 29, n. 2, p. 436-443, 2005.

STEIN, J. E.; GRECO, D. S. Portable blood glucose meters as a means of monitoring blood glucose concentrations in dogs and 
cats with diabetes mellitus. Clinical Techniques in Small Animal Practice, v. 17, n. 2, 70-72, 2002.

TILLEY, L. P.; SMITH, F. W. K. Apêndice I: Valores referenciais normais dos testes laboratoriais. Tabela I-B: Valores bioquímicos normais. In:__. Consulta veterinária em 5 minutos: espécies canina e felina. 2. ed. São Paulo: Manole, 2003. 1325 p.

WESS, G.; REUSCH, C. Evaluation of five portable blood glucose meters for use in dogs. Journal of the American Veterinary Medical Association, v. 216, n. 2, p. 203-209, jan. 2000a.
WESS, G.; REUSCH, C. Capillary blood sampling from the ear of dogs and cats and use os portable meters to measure glucose concentration. Journal of Small Animal Practice, v. 41, p. 60-66, 2000 b.

WEITGASSER, R.; GAPPMAYER, B.; PICHLER, M. Newer portable glucose meters-analytical improvement compared with previous generation devices? Clinical Chemistry, v. 45, n. 10, p. 1821-1825, 1999.

WIENER, K. Potential for error from underfilling Glucometer Elite test strips. Diabetic Medicine, v. 17, p. 555-556, 2000.

Protocolado em: 5 abr. 2008. Aceito em: 11 maio 2010 . 